\title{
Is Verification Getting Too Complex?
}

\author{
Yoav Hollander \\ Cadence Israel
}

\begin{abstract}
Verification of HW and HW/SW systems is becoming more and more complex. This presentation will look at the reasons for the increased complexity and what can be done about it.

Specifically, I shall look at where bugs come from and how they flow through the system (from specification, through design, detection, debug and removal). I shall then present some promising directions for reducing verification complexity, many of them attacking "incidental complexity".

Throughout the presentation, I shall try to emphasize currently-neglected research areas.
\end{abstract}

